\title{
Inhaltsverzeichnis und Gliederung
}

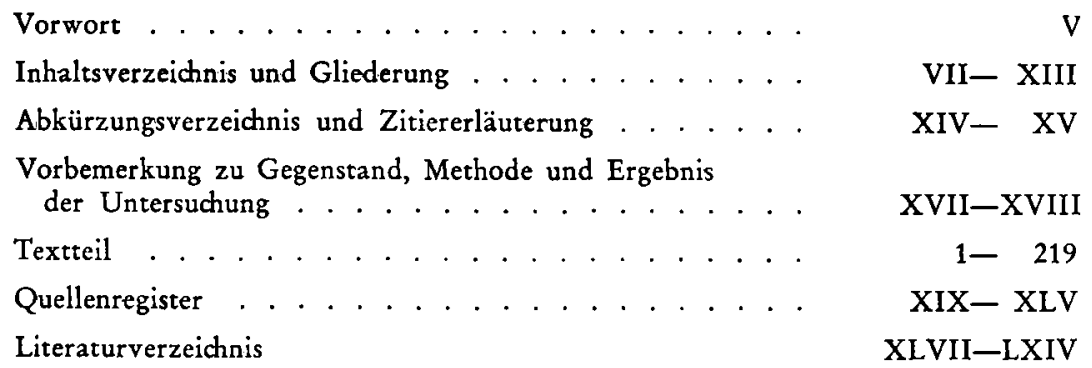

A. ERSTER TEIL:

Sachteil und Zubehör im römischen Recht 7

I. Der römische Sachbegriff. . . . . . . . . . . . . . . . . . . . 7

1. Der Sachbegriff als Begriff des sachenrechtsfähigen Gegenstandes . 7

a) Der Sachbegriff in der römischen Rechtssprache . . . . . . 7

b) Methodische Folgerungen ... . . . . . . . . . 10

2. Abgrenzung der sachenrechtsfähigen von den nicht sachenrechtsfähigen Gegenständen . . . . . . . . . . . . . . 11

a) Die unbeschränkt sachenrechtsfähigen Gegenstände . . . . . . 11

a) Lebende Körper (res se moventes): Sklave und Vieh . . . 11

B) Tote Körper (res mobiles) . . . . . . . . . . . . . 11

y) Liegenschaften (res immobiles): Grundstück (mit Aussaat,
Pflanzung, Haus) . . . . . . . . . . . . . 12

ס) Summenquanten (Münzen, Getreide) . . . . . . . . . . 12

b) Die Gegenstände mit beschränkter oder zweifelhafter Sachenrechtsfähigkeit ................ . . 12

c) Die nicht sachenrechtsfähigen Rechtsgegenstände . . . . . . 13 
3. Körperlichkeit des Gegenstandes als Kriterium unbeschränkter Sachenrechtsfähigkeit. Geltungsbereich und Bedeutung dieses

a) Beschränkung der Sachenrechtsfähigkeit auf wirtschaftlich nutzbare Sachkörper . . . . . . . . . . . . . . . .

b) Individualität und Integrität als Grenzbegriffe der Kürperlichkeit

a) Individualität . . . . . . . . . . . . . . 18

B) Integrität . . . . . . . . . . . . . . . . . 19

II. Der integrierende Bestand sachenrechtsfäbiger Einbeiten und deren integrale Binnenstruktur. Die drei Integrationsstufen.

1. Die erststufigen Integrate . . . . . . . . . . . . . . . 23

a) Rechtliche Eigenschaften . . . . . . . . . . . . . 23

b) Entstehungsgründe und betroffener Gegenstandsbereich . . . . 24

a) Die lebenden Körper . . . . . . . . . . . . . . . 24

B) Das Grundstück. satio, plantatio, aedificatio . . . . . . . 25

y) Die unteilbaren toten Individualsachen. Stoffquanten . . 25

8) Summenquanten . . . . . . . . . . . . . . . . . 26

c) Rechtsfolgen der Verbindung mehrerer Einzelsachen zu einem erststufigen Integrat .............. 26

a) Die Individualsachen (einschließlich Grundstücke) . . . . 27

$\beta$ ) Die Stoffquanten und Summenquanten . . . . . . . 28

2. Die zweitstufigen Integrate ............... 30

a) Rechtliche Eigenschaften . . . . . . . . . . . . 30

b) Entstehungsgründe . . . . . . . . . . . . . . . . . . 31

c) Betroffener Gegenstandsbereich . . . . . . . . . . . . . 31

a) Allgemeines . . . . . . . . . . . . . . . . . . . 31

B) Einzelüberblick . . . . . . . . . . . . . . 32

$\alpha a)$ Grundstücksverbindungen (vincta fixaque) . . . . . 32

$\beta \beta$ ) Gebäudeverbindungen . . . . . . . . . . . 35

(1) Die zwei Bestandteilsgruppen: (ergänzende und notwendige Bestandteile) ......... . 35

(2) Die objektiven Integrationsgründe (Festigkeit oder Notwendigkeit der Verbindung. perfectio integri) . $\quad 37$

(3) Die subjektiven Integrationsgründe (appositio ad usum perpetuum) . . . . . . . . . . . . . 38 


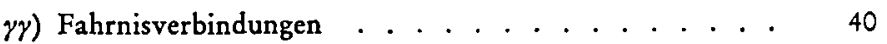

(1) Denkmal ............ . . . 4 40

(2) Schiff . . . . . . . . . . . . . . . . . 41 4

(3) Sonstige Fahrnis . . . . . . . . . . . 43

7) Zusammenfassung . . . . . . . . . . . . . 44

d) Rechtsfolgen der Verbindung mehrerer Einzelsachen zu einem zweitstufigen Integrat . . . . . . . . . . . . . 45

a) Der Rechtsstatus des Integrates . . . . . . . . . . 46

B) Der Rechtsstatus der Bestandteile . . . . . . . . . . . 49

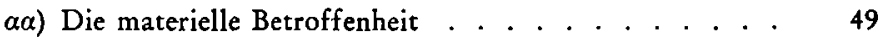

(1) Eigentumsstatus ........... . . 50

(a) Der Ausnahmefall (Eigentumsübergang auf den Eigentümer der Gesamtsache) . . . . . . . 51

(b) Die Regelfälle. actio de tigno iuncto und actio ad exhibendum ........... . 52

(c) dominium dormiens aut repristinandum? . . 53

(2) Bewegungsstatus ............ . . 55

(a) usucapio .............. 56

(b) vindicatio . . . . . . . . . . . . . 58

(c) Verfügungen ........... 60

$\beta \beta$ ) Die formelle Betroffenheit . . . . . . . . 62

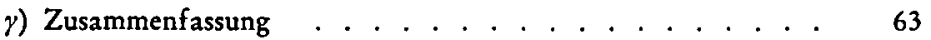

3. Die drittstufigen Integrate . . . . . . . . . . . . . . 64

a) Rechtliche Eigenschaften ................ . 64

b) Die Problematik positiver Existenz drittstufiger Integrate . . . 65

c) Ihr möglicher Gegenstandsbereich . . . . . . . . . . . 70

a) Mehrheiten gleichartiger lebender Einzelsachen (grex. familia, chorus, quadriga etc.) . . . . . . . . . . . 70

B) Mehrheiten ungleichartiger Einzelsachen, die den Komplex eines (werbenden) Grundstüdksinventars bilden (instrumentum) . . . . . . . . . . . . . . 71

y) Sonstige Sachmehrheiten (suppellex, bibliotheca etc.) . . . 71

III. Funktionale Sacheinbeiten obne Sachenrechtsfäbigkeit . . . . . . . . 72

1. Gegenstandsbereich .. . . . . . . . . . . . . 72

a) Die kleineren Einheiten . . . . . . . . . . . . . . . 72

b) Das Unternehmen . . . . . . . . . . . . . . . . . . . . 73

IX 
2. Rechtliche Behandlung .............. . 74

a) Allgemeines . . . . . . . . . . . . . . . . . . . 74

b) fundus cum instrumento . . . . . . . . . . . . . . 76

c) Praktische Auswirkungen der Sachenrechtsunfähigkeit. Rechtslagevergleich sachenrechtsfähiger und nicht sachenrechtsfähiger Einheiten .............. 78

IV. Die Erscheinungsformen und Entstebungsgründe von Sacheinbeiten im römischen Recht (Folgerungen aus II und III)

1. Die sachenrechtsfähigen Einheiten (Integrate) . . . . . . . 80

a) Erscheinungsformen . . . . . . . . . . . . . 81

a) Das erststufige Integrat . . . . . . . . . . . 81

B) Das zweitstufige Integrat . . . . . . . . . . . . 81

7) Das drittstufige Integrat . . . . . . . . . . . 82

b) Entstehungsgründe . . . . . . . . . . . . . . 82

a) Das erststufige Integrat . . . . . . . . . . . 82

B) Das zweitstufige Integrat . . . . . . . . . . 83

y) Das drittstufige Integrat . . . . . . . . . . . 83

2. Die nicht sachenrechtsfähigen Sacheinheiten 84

\section{B. ZWEITER TEIL:}

Sachteil und Zubehör im gemeinen Recht und in den Landesrechten seit dem Ende des 18. Jahrhunderts

I. Die Institutionengeschichte des Bestandteils- und Zubebörrechts . .

1. Pertinenz und Zubehör . . . . . . . . . . . . . . . . . . . 89

a) Die Naturrechtsgesetzbücher . . . . . . . . . . . 90

a) Das Instrument als Zubehör . . . . . . . . . . . . . . 90

B) Rechtsstatus des Zubehörs . . . . . . . . . . . . . . . 92

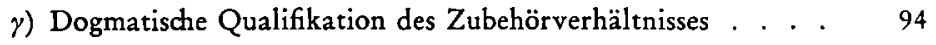

b) Das Pandektenrecht . . . . . . . . . . . . . . . . . . . 97

a) Grundsätzliches (Die Verbindlichkeit der römischen Tradition) ..................... 97 
B) Ausnahmen (Instrumentalsachen als Bestandteil oder Pertinenz) ................. aa) Instrumentalsachen als Bestandteil (untrennbare Verbindung) .............. . . . 100

$\beta \beta$ ) Instrumentalsachen als Pertinenz (Zwedkwidmung) . . 106

r) Rechtsstatus der Pertinenz . . . . . . . . . . . . . . 112

c) Die Gesetzgebung des 19. Jahrhunderts . . . . . . . . . . . 116

$\alpha$ ) Die Hypothekengesetze und Zivilgesetzbücher bis zum BGB 116

ק) BGB und ZGB. Ergebnis der Entwicklung . . . . . . . . 120

y) Die Executionsgesetze . . . . . . . . . . . . . 122

2. Bestandteil . . . . . . . . . . . . . . . . 123

a) Schrumpfung des integrierenden Bestandes (Ausscheidung des Zubehörs, Verselbständigung erststufig integrierender Grundstüdksbestandteile) . . . . . . . . . . . . . . . . 124

a) Ausscheidung der Pertinenzen . . . . . . . . . . . . . 124

B) Verselbständigung erststufig integrierender Grundstücksbestandteile ................. . . 126

aa) Das ALR . . . . . . . . . . . . . . . 126

$\beta \beta$ ) Code civil und ABGB . . . . . . . . . . . . . . 127

$\gamma \gamma)$ Die spätere Entwidklung . . . . . . . . . . . . . 127

b) Verdichtung des integrierenden Bestandes (Verwandlung zweitstufig integrierender in erststufig integrierende Sachen. Angleichung der $\mathrm{zweitstufigen}$ an die erststufige Integration) . . 129

a) Ursprünge der Entwidklung (Donellus) . . . . . . . . . 129

B) Die Naturrechtsgesetzbücher . . . . . . . . . . . . . 130

y) Die Pandektistik . . . . . . . . . . . . . . . . . . . 132

d) Die Gesetzgebung des 19. Jahrhunderts. BGB und ZGB . . 137

$\alpha \alpha$ ) Gebäudebestandteile . . . . . . . . . . . . . . . 138

$\beta \beta$ ) Grundstüdksbestandteile . . . . . . . . . . . . . 138

$\gamma \gamma$ ) Die integrierten Instrumentalsachen (Gewerbeausrüstung) 139

(1) Zürich, Bayern, Deutsches Reich (BGB) . . . . . 139

(2) Sachsen, Hessen, baltische Länder . . . . . . . . 140

(3) Schweiz (ZGB) 140

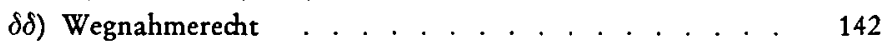

$\varepsilon \varepsilon)$ Die Fahrnisverbindungen . . . . . . . . . . 145

3. Das Ergebnis der institutionengeschichtlichen Entwidklung . . . 146

a) Die erststufigen Integrate . . . . . . . . . . . . . . . . 146

b) Die zweitstufigen Integrate . . . . . . . . . . . . . . . . 147 
c) Die Pertinenzen (das Zubehör) . . . . . . . . . . . . . . 148

d) Gesamtwürdigung und Ausblick . . . . . . . . . . . . . . 149

II. Die Theoriegeschichte des Bestandteils- und Zubebörbegriffs vom Naturrecht des 18. Jabrhunderts bis zum $B G B$

1. Die Naturrechtslehre

a) Die Theoriegeschichte bis zu Hofacker

b) Carl Christoph Hofacker (Das Integrationsprinzip der perfectio integri)

a) Die coordinativ-integrierende und die subordinativ-accessorische Sachverbindung . . . . . . . . . . . 156

ק) Hauptsache und Nebensache . . . . . . . . . . . . . 157

\%) Gesamtsache und Teilsache . . . . . . . . . . . 159

$\delta)$ Die korrespondierenden Erwerbsgründe accessio und specificatio . . . . . . . . . . . . . . . . 159

ع) Vergleich mit dem römischen Recht . . . . . . . . . . . 160

ac) Die Integritätsbestimmung . . . . . . . . . . . . . 161

$\beta \beta)$ Die Accessorietätsbestimmung . . . . . . . . . . 162

y.) Perspektiven . . . . . . . . . . . . . . . . 162

2. Die Naturrechtsgesetzbücher . . . . . . . . . . . . . . . . . 163

a) Das preußische Allgemeine Landrecht . . . . . . . . . 163

a) Der Substanzteilbegriff . . . . . . . . . . . 163

B) Der Pertinenzbegriff 164

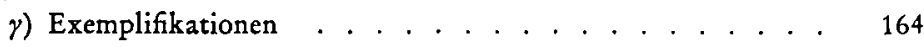

ס) Der Pertinentialstatus . . . . . . . . . . . 166

b) Das österreichische Allgemeine Bürgerliche Gesetzbuch und der französische Code civil . . . . . . . . . . . . . . 167

a) Das ABGB . . . . . . . . . . . . . . . . . . . . 167

B) Der Code civil . . . . . . . . . . . . . . . . . . 168

3. Die Pandektistik sowie Gesetzgebung und Spruchpraxis im 19. Jahrhundert . . . . . . . . . . . . . . . . . 169

a) Uberblick . . . . . . . . . . . . . . . . . . . . . . 169

b) Der undifferenzierte, ältere Pertinenzbegriff der Frühpandektistik . . . . . . . . . . . . . . . . 175

c) Franz Gesterding (Das Integrationsprinzip der perfectio integri) 179

d) Gottlob Leberecht Funke (Das Integrationsprinzip der perfectio integri aut integritas partium) ............. 181 
e) Carl Georg von Wächter (Das Integrationsprinzip der corporalis cobaesio in perpetuum facta) . . . . . . . . . . .

a) Bestandteilsbegriff und Pertinenzbegriff. Die Körperlichkeit der Verbindung als integritätsbegründendes Prinzip . . . . 184

B) Rechtsstatus von Bestandteil und Pertinenz . . . . . . . 186

y) Perspektiven . . . . . . . . . . . . . . . . . . 187

f) Das Integrationsprinzip der mera corporalis cobaesio nach Wächter . . . . . . . . . . . . . 188

a) Die Wissenschaft . . . . . . . . . . . . . 188

B) Die Gesetzgebung . . . . . . . . . . . . . . . . 194

g) Georg Friedrich Puchta (Das Integrationsprinzip der necessitas coniunctionis ... . . . . . . . . . . . . . 195

a) Der systematische Ansatz (Teilbarkeit) . . . . . . . . . 196

B) Wesentliche und nicht-wesentliche Teile . . . . . . . . . 197

$\gamma$ ) Die Notwendigkeit der Verbindung als einheitliches, integritätsbegründendes Prinzip . . . . . . . . . . . . . . . 199

ס) Der Rechtsstatus des Bestandteils . . . . . . . . . . . . 200

ह) Die Pertinenzen . . . . . . . . . . . . . 201

h) Das Integrationsprinzip der necessitas coniunctionis nach Puchta 202

a) Die Wissenschaft (necessitas coniunctionis) . . . . . . . 202

$\beta$ ) Die Wissenschaft, Fortsetzung (wesentlicher und unwesentlicher Bestandteil) . . . . . . . . . . . . . 207

y) Die Rechtsprechung . . . . . . . . . . . 210

ס) Die Gesetzgebung . . . . . . . . . . . 210

4. Das Ergebnis der theoriegeschichtlichen Entwicklung . . . . . . 215

SCHLUSSBETRACHTUNG 218 